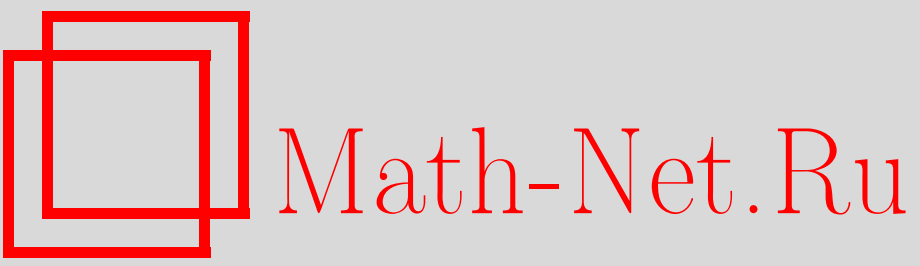

Ф. М. Мухамедов, О бесконечномерных квадратичных вольтерровских операторах, УМH, 2000, том 55, выпуск 6, 149-150

DOI: https://doi.org/10.4213/rm349

Использование Общероссийского математического портала Math-Net.Ru подразумевает, что вы прочитали и согласны с пользовательским соглашением

http://www.mathnet.ru/rus/agreement

Параметры загрузки:

IP: 44.207 .124 .84

26 апреля 2023 г., 04:20:34 


\title{
О БЕСКОНЕЧНОМЕРНЫХ КВАДРАТИЧНЫХ ВОЛЬТЕРРОВСКИХ ОПЕРАТОРАХ
}

\author{
Ф. М. Мухамедов
}

Понятие квадратичного стохастического оператора впервые было сформулировано в работе С.Н. Бернштейна [1]. Одной из задач при изучении квадратичных операторов является исследование эргодических свойств таких операторов [2], [3]. Одним из классов квадратичных операторов является класс вольтерровских операторов, изучению которого посвящены многочисленные работы (см., например, [4]).

В статье дается определение бесконечномерного квадратичного вольтерровского оператора и изучаются некоторые свойства траекторий таких операторов.

Через $S$ обозначим следующее множество:

$$
S=\left\{x=\left(x_{n}\right): x_{i} \geqslant 0, \sum_{n=1}^{\infty} x_{n}=1\right\} .
$$

Ясно, что $S$ является выпуклой оболочкой векторов вида

$$
e^{(n)}=(0,0, \ldots, 1,0, \ldots),
$$

где единица стоит на $n$-м месте, и только они являются экстремальными элементами множества $S$. Множество экстремальных точек $S$ обозначим через $\operatorname{extr}(S)$.

Определим оператор $V: S \rightarrow S$ следующим образом:

$$
(V(x))_{k}=\sum_{i, j=1}^{\infty} p_{i j, k} x_{i} x_{j}, \quad k \in \mathbb{N}, \quad x=\left(x_{i}\right) \in S,
$$

где

$$
p_{i j, k} \geqslant 0, \quad p_{i j, k}=p_{j i, k}, \quad \sum_{k=1}^{\infty} p_{i j, k}=1, \quad i, j \in \mathbb{N} .
$$

Так определенный оператор будем называть бесконечномерным квадратичныц стохастическим оператором. Оператор $V$, определенный равенством (1), называется вольтерровским, если $p_{i j, k}=0$ при $k \neq i, j$.

Для $x, y \in S$ обозначим

$$
(\widetilde{V}(x, y))_{k}=\sum_{i, j=1}^{\infty} p_{i j, k} x_{i} y_{j}, \quad k \in \mathbb{N} .
$$

Напомним, что выпуклое множество $C \subset S$ называется гранью, если из того, что $\lambda x+(1-$ $\lambda) y \in C$, где $x, y \in S$ и $\lambda \in(0,1)$, следует $x, y \in C$. Совокупность всех граней множества $S$ обозначим через $\Gamma$. Для векторов $\varphi, \psi \in S$ обозначим $\Gamma(\varphi, \psi)=\{\lambda \varphi+(1-\lambda) \psi: \lambda \in(0,1)\}$.

ПредЛОЖЕНИЕ 1. Квадратичный оператор $V$ вольтерров тогда и только тогда, когда для любъх $\varphi, \psi \in \operatorname{extr}(S)$ имеет место $\widetilde{V}(\varphi, \psi) \in \Gamma(\varphi, \psi)$.

Через $Q V$ обозначим совокупность всех вольтерровских операторов. Наделим $Q V$ топологией, которая определяется по сходимости: сеть $V_{\nu}$ сходится к $V$, если для любого $x \in S$

$$
\lim _{\nu \rightarrow \infty}\left(V_{\nu}(x)\right)_{k}=(V(x))_{k}, \quad k \in \mathbb{N} .
$$

ПреДЛОЖенИЕ 2. $Q V$ является выпуклым слабо компактным множеством.

Из этого предложения и в силу теоремы Крейна-Мильмана следует, что множество всех экстремальных вольтерровских операторов $\operatorname{extr}(Q V)$ непусто.

Квадратичный оператор $V$, определенный равенством (1), называется чистым, если для всех $\varphi, \psi \in S$ имеет место

$$
\widetilde{V}(\varphi, \psi) \in \operatorname{extr} \Gamma(\varphi, \psi) .
$$

Ясно, что чистые квадратичные операторы являются воль терровскими. 
Теорема 1. Квадратичный оператор $V$ чистый тогда и только тогда, когда он экстремальный в $Q V$, m.е. $V \in \operatorname{extr}(Q V)$.

ПрЕДЛОЖЕниЕ 3. Пусть $V \in Q V-$ вольтерровский оператор, тогда $x^{\prime}=V x$ можно записать в виде:

$$
x_{k}^{\prime}=x_{k}\left(1+\sum_{i=1}^{\infty} a_{k i} x_{i}\right), \quad k \in \mathbb{N},
$$

əде $a_{k i}=-a_{i k},\left|a_{k i}\right| \leqslant 1$.

СледСтвиЕ 1. Любая грань симплекса $S$ инвариантна относительно действия вольтерровского оператора $V$.

СлЕДСТвиЕ 2. Вершины симплекса, т.е. грани $\Gamma_{I}$ при $|N \backslash I|=1$, являются неподвижными точками вольтерровского оператора $V$.

В конечномерном случае известно (см. [4]), что каждый вольтерровский оператор является биективным отображением симплекса. С помощью предложения 3 доказывается следующая

Теорема 2. Пусть $V \in Q V$ - вольтерровский оператор, тогда он является автоморфизмом $S$.

Пусть $V: S \rightarrow S$ - вольтерров оператор, т.е.

$$
x_{k}^{\prime}=x_{k}\left(1+\sum_{i=1}^{\infty} a_{k i} x_{i}\right), \quad k \in \mathbb{N},
$$

где $a_{k i}=-a_{i k},\left|a_{k i}\right| \leqslant 1$.

Через $\mathscr{G}$ обозначим множество решений системы неравенств

$$
\sum_{i=1}^{\infty} a_{k i} y_{i} \leqslant 0, \quad k \in \mathbb{N},
$$

принадлежащих $S$.

ЗАмечАниЕ. Известно [4], что в конечномерном случае множество $\mathscr{G}$ всегда является непустым множеством. В бесконечномерном случае, в отличие от конечномерного, множество $\mathscr{G}$ может оказаться пустым.

ПримеР. Рассмотрим вольтерровский оператор, определенный следующим образом:

$$
V_{0}:\left\{\begin{array}{l}
x_{1}^{\prime}=x_{1}\left(1-x_{1}\right), \\
x_{k}^{\prime}=x_{k}\left(1+x_{k-1}-x_{k+1}\right), k \geqslant 2,
\end{array}\right.
$$

тогда для этого оператора множество $\mathscr{G}$ пусто.

Далее предположим, что множество $\mathscr{G}$ непусто. Тогда ясно, что $\mathscr{G}$ выпуклое подмножество $S$ и состоит из неподвижных точек оператора $V$.

Теорема 3. Пусть $V$ - вольтерров оператор, и пусть $x^{0} \in S$ такова, что $x_{i}^{0}>0$, $\forall i \in \mathbb{N}$. Если $V x^{0} \neq x^{0}$ и предел $\lim _{n \rightarrow \infty} V^{n} x^{0}$ существует, тогда предельная точка траектории лежит в $\mathscr{G}$.

\section{СПИСОК ЛИТЕРАТУРЫ}

[1] Бернштейн С. Н. // Уч. зап. Н.-И. каф. Укр. отд. мат. 1924. № 1. С. 83-115. [2] Kesten H. // Adv. Appl. Probab. 1970. V. 2. Р. 1-82; С. 179-228. [3] Любич Ю. И. // УМН. 1971. Т. 26. № 5. С. 51-116. [4] Ганиходжаев Р. Н. Квадратичные стохастические операторы и функции Ляпунова // Матем. сб. 1992. Т. 183. № 8. С. 119-140.

Принято редколлегией 03.10 .2000 